\title{
Photonics Applications and Web Engineering WILGA May 2013
}

\author{
Ryszard S.Romaniuk \\ Warsaw University of Technology, Institute of Electronic Systems, Poland
}

\begin{abstract}
Traditional WILGA Symposia are held two times a year since 1998. Each year the WILGA May edition gathers around 300 young researchers active in advanced photonics and electronics systems. The paper, as each year, presents a digest of chosen technical work results shown by young researchers from different technical universities from this country during the SPIE-IEEE Wilga 2013 Symposium on Photonics and Web Engineering. Topical tracks of the symposium embraced, among others, nanomaterials and nanotechnologies for photonics, sensory and nonlinear optical fibers, object oriented design of hardware, photonic metrology, optoelectronics and photonics applications, photonics-electronics codesign, optoelectronic and electronic systems for astronomy and high energy physics experiments, CMS, ITER, JET Joint European Torus, BRITE nanosatellite, and pi-of-the sky experiments development. The symposium is an annual summary in the development of numerable Ph.D. theses carried out in this and neighboring countries in the area of advanced electronic and photonic systems. It is also a great occasion for SPIE, IEEE, OSA and PSP students and young researchers to meet together in a large group spanning the whole country with guests from this part of Europe.
\end{abstract}

Keywords: nanomaterials, optical fibers, optoelectronics, photonics, measurement systems, astronomy, high energy physics experiments

\section{INTRODUCTION}

This paper is a digest of chosen presentations from annual meeting of young science in Poland. The 32nd Symposium of young scientists WILGA 2013 on Photonics and Web Engineering has gathered around 300 participants [1]. There were presented around 200 papers - mainly concerning the realized Ph.D. theses and participation in research projects relevant to the topical area of the meeting. There were also presented a few plenary papers introducing the audience into new research areas of photonics and electronics. The symposium is organized under the auspices of the SPIE - The International Society for Optical Engineering, IEEE (Poland Section and Region 8), Photonics Society of Poland, KEiT PAN, PKOpto SEP and WEiTI PW. The Symposium is supported by a number of large European projects, realized inside the FP7 framework. This year the projects were: EuCARD - European Coordination for Accelerator R\&D, and TIARA-PP - Test Infrastructure and Accelerator Research Area - Preparatory Phase. A special session was held on these projects during WILGA 2013. The symposium is organized annually by young researchers from the PERG/ELHEP Laboratory of ISE PW with cooperation of SPIE and IEEE Student Branches. Media patronage over the symposium is extended by Elektronika monthly technical magazine. Symposium proceedings are published by Elektronika by SEP - Association of Polish Electrical Engineers, JET - Journal of Electronics and Telecommunications KEiT PAN and Proceedings SPIE [2-4]. Wilga Symposium is topically associated with the cyclic research meetings on optical fibers and their applications organized in Bialowieza and Naleczow (UMCS Univ., Lublin) every 18 months. There are presented in this paper some work results from the main of the most interesting sessions or topical tracks of WILGA 2013 Symposium. WILGA Symposium will be continued in 2014, as usual, in January and May editions. This paper is the first part from Wilga 2013 May edition concentrating on optoelectronics and computational intelligence.

\section{TOPICAL TRACKS OF WILGA 2013}

The topical session and tracks of WILGA 2013 were as follows: microwave and THz measurements, solving of inverse problems in microwave and photonics technology, nanotechnologies and nanomaterials for optoelectronics and photonics, optical fibers for sensors and all-photonic devices for sensors, active optical fibers, sensors and sensory networks, object oriented design of optoelectronic and photonic hardware, photonics applications, advanced bioelectronics and bioinformatics, co-design of hybrid photonic - electronic systems, computational intelligence in optoelectronics and robotics, development in the wide-angle astronomic observations of the whole sky - pi-of-the-sky project, processing and imaging of multimedia data streams, machine vision, vehicles - quadrocopter and Mars rover, analog transmission systems in noisy conditions with digital reverse transmission channel, optoelectronic and photonic

Photonics Applications in Astronomy, Communications, Industry, and High-Energy Physics

Experiments 2013, edited by Ryszard S. Romaniuk, Proc. of SPIE Vol. 8903, 890303

(C) 2013 SPIE · CCC code: $0277-786 X / 13 / \$ 18 \cdot$ doi: $10.1117 / 12.2035420$ 
metrology, reconfigurable measurement systems, high performance - low-jitter low-latency transmission systems White Rabbit and atomic clocks, thermonuclear fusion experiments - JET and ITER, research results update from HEP experiments - TOTEM and CMS/LHC in CERN. A number of Wilga sessions concerned applications aspects of photonic and electronic circuits and systems, including advanced applications which combine hardware and software. A separate session track was organized by SPIE, IEEE, OSA and PSP - Photonics Society of Poland students for the new students beginning their adventure with the science of photonics and electronics.

\section{OPTOELECTRONICS AND PHOTONICS}

Fiber optic microphone was researched based on Sagnac interferometer (ISE and IT PW). Sensory applications of Sagnac interferometers have one large advantage. The construction is relatively simple. There is required only one half/half coupler, source, detector and polarization maintaining fiber loop. A strand of optical fiber in the loop is attached to a loudspeaker. Characteristics, in time and frequency domains, of such a microphone were presented.

Optoelectronics Laboratory at ISE PW (Warsaw Uni. Technology) specializes in design, manufacturing, characterization and applications of optical fiber Bragg gratings. Tapered OFBG are used to obtain the effect of optical chirp, where the Bragg wavelength is a function of longitudinal coordinate, because the effective refraction changes with the tapered fiber length. The changes are of the order of $10^{-3}$. As a result the fiber core power ratio changes with $\mathrm{z}$ coordinate. Analyzing this effect, coupled mode theory was applied. Solving CMT equations leads to determination of elements in the Transfer Matrix of this photonic system. In Transfer Matrix Method a uniform Bragg grating can be represented in matrix form. Matrix elements/coefficients are representing particular transmittance effects like: DC self coupling, AC coupling, detuning, DC coupling, visibility, amplitude of the core refractive index change. Usage of TMM to tapered OFBG requires division of the optical two-port to relevant concatenated uniform OFBG - sections. Each section has different but assumed as constant detuning parameter, different Bragg wavelength and DC self coupling coefficient. The resulting optical transmittance is a product of section transmittances. The cone lengths of the analyzed photonic components were of the order of $25 \mathrm{~mm}$. Core dimensions were in the range of 3,5-6,5 um. Tapered OFBG characteristics, like transmittance and/or reflectivity are tuned by its length and taper parameters. Complex photonics Bragg structures can be designed efficiently using either coupled modes or matrix transmittance methods.

Shaping the characteristics of OFBG is done by several methods, including refractive, phase, geometrical and others. One of the methods is weak or strong tilting - slanting. Even more complex characteristics are obtained by combining several methods like tilting with chirping. Potential applications of tilted OFBG include: gain flattening filter in EDFAs, narrow band filters, add/drop optical MUX, chemical sensors of increased sensitivity, mechanical sensors - including bend, twist, strain, compression; spectrometers, refractometers, polarimeters, amplitude discriminator in fiber multiplexed sensor systems for wide spectral range, and modal power distribution meters. One of the manufacturing method of titled OFBG is introducing the required tilt angle of the phase mask against the fiber axis. Characteristic parameters of the tilted OFBG are: grating period resulting from the interference pattern, and tilt grating period along the optical fiber axis. The introduced tilts were of the order of single degrees. The tilt shifts the characteristic to shorter wavelengths, widens it to a form of multiple spikes - oscillations arranged to a wide envelope. Only small part of the original un-tilted characteristic of the same OFBG remains un-shifted.

All parameters of optical fibers, OFBG and photonic components compatible with optical fiber transmission systems may be measured by a robotic measurement set up. The measurements include the following characteristics: spectral transmission, filter - reflection, sidebands, dispersion, refraction. The work is done in ISE PW in cooperation with Nat. Inst Telecommunications. The measurement system consists of: tunable optical source, broadband optical source, RF generator, optical modulator (for example M-Z interferometer), OSA, fiber optic components like splitters, circulators, optical and vector voltmeter. Lab View is conveniently used for measurement control and data processing. The system abilities was validated using the following photonic components: DSF fiber in the vicinity of zero dispersion wavelength, linearly chirped OFBG. In all cases the measurements were compared with theoretical calculations. The advantages of such universal measurement system are: simultaneous measurements of spectral transmission and/or reflection and dispersion parameters, high accuracy, flexibility and reconfiguration possibility, fast and highly standardized measurement due to automation. 
Laser based ion implantation is a very flexible tool used for surface modification. Laser ion source (LIS) is able to generate ion beams of nearly all solid materials. The issues associated with LIS technology are: ion beams are contaminated with other particles and other ions; the ions are not monochromatic in energy but have quite broad spectrum, the implantation depth is not well defined, deposition is often the dominating mechanism. LIS is used in combination with electrostatic ion acceleration, where groups of ions obtain the same energy of narrow distribution. Deflecting electrodes are used to filter out majority of contamination. LIS is used most frequently for deposition of thin films of certain composition. The works on LIS are carried out at IFPiLM Institute in Warsaw. Ion streams are generated out of solid material in two ways: by collision absorption involving inverse bremsstrahlung, cascade ionization, creation of plasma plume and plasma screening; and collision-less absorption, where high power laser is required inducting parametric instability in material. Both processes result in emission of particles including ions. The ions are then controlled by electromagnetic field. To control the process fully, and the LIS be operable, several quantities have to be measured like: temperature and energy of plasma components; distribution of plasma component concentrations, plasma characteristics and emitted radiation - charged particles, photons and uncharged particles. The used diagnostics methods are for ions, x-rays, VUV/IR photons, interferometry and polarimetry, Raman spectrometry, XPS depth profilometry and neutron diagnostics. Tests were done with Ge deposition on $\mathrm{SiO}_{2}$ substrate. The experiments show that laser induced plasma contains contaminations to be filtered out by electrostatic accelerating and deflecting filters. More accelerating chooses from plasma monochromatic ions. These ions are appropriate for implantation. Apart from ions nanocrystals are present in the plume and they may be chosen for deposition of target surface. LIS source with electrostatic filters and accelerators are tested for photonics applications, including: depositions of thin films at fiber face, implantation and/or deposition on fiber claddings for sensors, couplers and other photonic functional devices. It is also appropriate technique for plasmonic technologies and for building of fine nanostructures.

Silicon carbide $\mathrm{SiC}$ photodiodes, transparent in the visible range are researched for many applications including sensors, high temperature technologies, photovoltaics, and many others. The research is carried out in IMiO WUT (prof.M.Borecki). The capabilities of optoelectronic sensors can be extended by use of spectral data processing. A popular range of wavelengths that are used is from UV at $260 \mathrm{~nm}$, where: DNA absorbs radiation, to the visible, where the results can be directly observed by the human eye, Fire and explosion emits radiation to VIS and MIR. This wide range (UV-NIR) can be examined with a spectrophotometer, but in many situations the use of this kind of instrument is not desirable because of its cost and complexity. Sensing of a sample using one beam with different wavelengths at the same time is required when the sample is in movement or in a dynamical state, as happens in analyzing the sedimentation of a mixture. Using LEDs as light sources is advantageous because of their low cost and ease of electronic modulation which makes the sensor more immune to background radiation. That increases the optical power balance on the detector side enabling use of photodiodes instead of APD or PMT detectors. The LEDs radiation can be coupled to beam with a fiber optic bundle. There are a few ways of constructing the device that allows in one optical axis simultaneous detection of a UV and VIS radiation beam. First is the use of commercially available components, such as Si and Si UV enhanced photodiodes with dichroic mirrors. Second is the use of hybrid technology to build photodiode matrices that use $\mathrm{SiC}$ and $\mathrm{Si}$ components or $\mathrm{Si} \mathrm{UV}$ enhanced components and matrices of optical filters. Third possibility is the use of stack of detectors using one transparent-to-VIS radiation element that absorbs and detects UV radiation. Spectral sensing of bio-diesel-fuels in UV was performed. In order to bring the absorbance to the measurable range of a spectrophotometer, the samples were diluted with $\mathrm{n}$ - heptane. The dilution was completed in three steps. In each step, $0.7 \mathrm{~mL}$ of sample was accurately mixed with $9.3 \mathrm{~mL}$ of $\mathrm{n}$ - heptane. The final dilution of all biodiesel diesel blends was 1:2915 v/v. The LEDs with wavelength of characteristic absorption in UV are now accessible. Measurement procedure implementation in sensors is problematic due to $n$-heptane use which is flammable, harmful and dangerous to the nature.

The UV large area SiC photodiode transparent in VIS construction was investigated. The components had the following characteristics. The honey comb shape front contact offers advantages over classical approximation of upper electrode structure. The transparency of photodiode in VIS enables additional examination of signals in sensing applications. The responsivity of large area SiC photodiode in cuvette holder is the same as commercial one. The dark current is high and strongly depends on supply conditions because the structure was examined without the guard ring. The electric origin noise results in rejection of constant current examination of weak signals even for solar blind photodiodes. Low level UV signals under examination are typical in absorption measurements of bio-components in 
liquid. The large area $\mathrm{SiC}$ photodiode with integrated trans-impedance amplifier works better than photodiode in direct reverse configuration connected to amplifier at a distance. It results in: simpler DC noise filtering, and two times better noise coefficient $\mathrm{S} / \mathrm{N}$ even with application of a standard amplifier.

\section{ICAN PROJECT - OPTICAL FIBER MEGA-LASER}

Polish laser laboratories are expected to take part in the ICAN. ICAN project is to built a nearly 100 Joule optical fiber laser, oriented to novel applications. ICAN project concerns, among others, the potential usage of laser plasma wakefield acceleration of charged particles. The advantage is a promise for great field gradients going up to $300 \mathrm{GV} / \mathrm{m}$, i.e. $10^{4}$ times bigger than in current big accelerators. Or the reverse, the plasma wakefield accelerator can be $10^{4}$ times smaller to obtain potentially the same acceleration effects. To wake god quality fields in plasma a coherent laser is needed of huge power and very fast pulse repetition rate. The requirements are: peak laser power - a few PW, optical average power - MW, high energy conversion efficiency - not worse than $40 \%$. Very precise amplitude and phase control. Such a source can be done by assembling and optically phasing tens of thousands of high power optical fiber lasers. Single mode $\mathrm{Yb}$ doped fiber amplifier has optical output at the level of $\mathrm{mJ}$, but the output energy from the set-up may reach $100 \mathrm{~J}$, at pulse duration of $10 \mathrm{fs}$. The amplifier has several stages to multiply fiber output to tens of thousands. The system operates inside on stretched pulses by means of chirped gratings. At the system output the pulses are compressed again to the initial value and precisely phased. The basic block of the system is single mode optical fibers amplifier with double cladding, and pumped from the far end face by high power diode laser. Bragg gratings are integrated into the core of optical fiber amplifier. ICAN is a project realized inside the EU FP and chaired by prof.G.Mourou. Assembling huge Yb-fiber phase array has several advantages against other solution of lasers of comparable beam parameters: high efficiency of end face diode pumping, good cooling ability of fiber, fiber is resilient to thermal distortions, good beam quality, cheap and mass manufacturable. The issue to be solved is phasing of thousands of fibers, possibly grouped in smaller phased bunches. Single fiber output is $2 \mathrm{~mJ}$ pulse, $200 \mathrm{fs}, 800 \mathrm{~W}$ average power, at $40 \mathrm{kHz}$ repetition rate. Aggregated power/energy of $100 \mathrm{~J}$ at $50 \mathrm{fs}$ may be obtained from around $5^{*} 10^{4}$ of such fibers. Applications of such a source are very attractive: acceleration of $10 \mathrm{GeV}$ electrons and relativistic protons; TeV laser collider research; FEL infrastructure comparable to LCLS but with much higher pulse repetition bigger than $1 \mathrm{kHz}$; laser driven transmutation reactor; proton and/or carbon therapy; x-ray and gamma ray generation, etc.

\section{PHOTONICS/ELECTRONICS FOR HIGH ENERGY PHYSIC EXPERIMENTS, FUSION, AND SPACE/ASTRO EXPERIMENTS}

The main effort at the LHC centered experiments were focused at Higgs discovery. Higgs, in its standard scalar version belongs still to the SM. LHC ambition is also search for new physics, at which gates we now apparently are standing, with the measurement possibilities. The SM does not contain gravity, thus an extended model is needed. Dark matter and dark energy is far from any explanation. Neutrino nature is only known on its surface. There are several candidates for dark matter non-barionic particle, like WIMPs or HSCPs. The search for HSCPs (heavy pseudo-stable charged particles) is considered by the CMS detector using a modified RPC (resistive plate chamber) sub-detector, with modified trigger. Due to their mass, the HSCP may be recognized by their large latency, arriving not in their bunch crossing windows but respectively later. The search for new physics has several main research paths. Some of them are: dark matter particles, neutrinos, super symmetry - assuming the existence of heavy symmetric particles equal in number with fermionic and bosonic particles; technicolors - assuming Higgs boson as a complex particle of internal structure revealed at energies above $\mathrm{TeV}$; and more dimensions. The estimates for the searched HSCP are: they are lepto-quarks with mass over $150 \mathrm{GeV}$. Estimated lifetime, $75-105 \mathrm{~ns}$, is large enough to reach the outer layers of the CMS detector. Other particles of new physics to be searched are gluinos (mass $370 \mathrm{GeV}$ and lifetime $10-1000$ s excuded), stau - the supersymmetric partner of tau lepton. Search for HSCPs at RPC is based on the following assumptions: RPCs are sensitive to all charged particles, HSCP are expected to be highly ionizing; TOF of HSCP is much bigger than leptons; normalized velocity is 0,8 thus they may be identified as muons. Newly implemented RPC PAC algorithm now also enable recognition of HSCPs. Most of the HSCPs are expected to hit at the small angles, what stems from the muon movement analyses and simulations. The work on the upgrade of the CMS RPC system to enable detection of new particles is done by the Warsaw CMS Group.

A research group at ISE WUT is working on the next generation of plasma diagnostics for two tokomaks - JET and finally ITER. The system has to be multichannel and accept high input data rate, and complex data analysis algorithms 
implementation. The hardware is based on embedded mITX motherboard connected to multi-FPGA treelike topology. In the current system only processed data is available, while the research procedures need access to raw data. There is a need to store large amount of data via a nondependent communication channel with reliable and fast data transmission. The system consists of FPGA logic, GTP (SerDes) link, DDR3 memory and custom interface - which is suitable for diagnostic purposes. The system is to handle data overflow in a predicted and structured manner. GTP links handle up to $3.125 \mathrm{Gbps}$ data traffic and assure very low jitter. Low level, packet based Aurora protocol as applied, which handles GTP instantiation, synchronization, clocking, data validity signaling, and provides CRC checking. Modified Aurora IP core increases the efficiency of usage of GTP transceivers.

Polfel laser project, planned at NCBJ Swierk, requires additional work on beam dynamical simulations on superconducting cavity model. Two parameters were of special concern: emittance and multipacting. Emittance is an area in X-X' plane (position and angle) occupied by beam particles, divided by $\pi$. Emittance is one of the key parameters determining the overall performance of an accelerator, like luminosity of particle beams, brightness of synchrotron photon beams, saturation length in FEL undulator, and resolution of fixed target experiments. Emittance is the energy radiated by the surface of a body per second per unit area. Slice emittance is an emittance of a smaller part of radiating body inside the big one. Particle beam is modeled as a cylinder emitted from a cathode. Slice emittance is the one of a smaller cylinder inside the big one. Slice emittance is researched as function of different parameters. Slice emittance is observed due to differences between particle speeds in the front and the back parts of the bunch.

Multipacting is a phenomenon of resonant electron multiplication. A number of electrons build up an avalanche leading to considerable power loss in the bunch and heating of the walls of vacuum beam pipe. Multipacting electrons create a cloud preventing the increase of the cavity field by the increase of incident power. Increase of temperature in superconducting cavity leads to thermal breakdown and cavity quenching. Multipacting electrons bombard ceramic windows of the high power coupler, and at some intensity may break it leading to vacuum loss. They also bombard metal surfaces damaging them. Modeling of all these harmful effects is necessary for the design of optimal cavity.

Research Group from ISE WUT, IFPilLM and NCBJ Warsaw are working on a new generation of plasma diagnostic, magnet management, and general control-measurement systems serving laboratory, test and large tokomaks. These machines include MAST, JET and ITER. The major aim of experiments with tokomaks is future fusion energy production. The used reaction is $\mathrm{D}+\mathrm{T}=\mathrm{n}$ (high kinetic energy) $+\mathrm{He}$. Advantages of this reaction are: no emission, available fuel, extreme potential energy efficiency, relatively high safety as the reaction is not self supporting. The disadvantages are: high temperature (over $10^{8} \mathrm{~K}$ ), high neutron radiation, which is converted to electricity only indirectly, via heat production and heat diverting. Till now, not all problems with ignition and reaction maintenance for some short period of time were solved. A number of experiments around the World, including several of them in Europe are trying to master the fusion reaction. One of them is the UK's fusion energy experiment - the MAST. MAST is a Mega Ampere Spherical Tokamak. It is expected that designs either based on or using some of its idea and test results may lead to a compact Component Test Facility (Fusion CTF), which would reduce the risk and accelerate the development of fusion power. MAST facility is now under development and modernization. The expected functionalities of diagnostic and management system for MAST (magnets) are a follows: managing of current and voltage in critical system sections, monitoring current and voltage in critical systems sections, provide fat magnet discharge, other features. The system architecture is as follows: MAST magnets, sensors, E/O converters, optical fiber links, SFP - FMC, AMC PCB, backplane, $\mu$ TCA controller and hub, carrier management, MCH PCIe switch, CPU, and Ethernet. The $\mu$ TCA carrier is a very efficient, highly standardized hardware platform for measurement, control and intensive - heavy duty information processing, and generally for hardware - software system co-development. The carrier management system uses IPMI standard of intelligent platform management. High speed communication is available in the backplane like GbE and PCIe. It features high degree of modularity, adding a lot of flexibility for the design. IPMI features: power management, temperature monitoring, hot swap handling, monitoring of system components state and health. A relevant AMC card collection with optical links is under development with magnet management functionalities. Optical link features: SMA/BNC connector, amplifier, DAC/ADC, buffers, MUX, DIO, TLK2501, SFP socket, SFP modules, dual fibre cables, UFL connectors and FMC connector. Optical ADC - DAC is also under design. IPMI and AMC card management was implemented on Cortex-M3.

A subject of design is high speed analog front end for GEM detector. The Gas Electron Multiplier Detector is composed of: drift cathode, micro perforated GEM foil, and readout board. GEM detector works as follows (in simplification). 
Ionizing particles enter the detector via the drift cathode and collide with gas particles. Free electrons are pulled into GEM foil's holes. Due to avalanche effect, the electrons are multiplied and directed to the readout board. Electrons generate (induce) charge on the electrodes of the readout board. A typical DAQ method was applied consisting of the following stages: GEM, amplification, signal shaping, ADC, FPGA processor. The detector has large amplification and deals with a lot of ionizing particles. The resulting pulses in the detector are short. Components in the signal processing channel have also to be fast, including charge amplifier, shaping module and ADC. Using fast ADC in all readout channels involves much more costs. ADC consume a lot of power. For each row and column of GEM there is needed one ADC, thus for the matrix 128x120, there are needed 256 ADCs. A better solution is to replace the shaping module and shift integration from FPGA to an analog circuit - analog integrators with different kinds of discharging mechanism. This solution needs mitigation of saturation effects.

Large accelerator complex FAIR is under construction at GSI Helmholtz in Darmstadt. The PERG/ELHEP Group is designing software for beam trajectory measurement system. The used hardware platform is uTCA crate, custom AMC FMC carriers, custom FMC ADC cards, AMC card with Intel core 17 processor running custom software and Linux OS. The assumptions/requirements are: software based on Linux OS, fast and reliable, written in C++ with Boost and STL libraries, featuring parallel computing multi-process software, able to work with custom hardware. Predicted software architecture is: multiple class, object programming based, complicated, and of flexible architecture. The data flow in software architecture is: FMC carrier, PtoR Calc, Dynamic storage in shared memory, RtoH, Sync, Diag, Init, Nonvolatile storage, GUI and finally user. The processes, centered at SYNC include: RtoH, Calc, Init, Diag and PtoR. The system design is in progress.

A Research Group specializing in detectors and front-end electronics for HEP experiments and neural network based data selection algorithms is active in IRE WUT (prof.K.Zaremba, prof.J.Marzec). The Group participates in COMPASS experiment in CERN and in T2K experiment in Japan in cooperation with NCBJ Świerk. Detector construction for COMPASS included: straw tube chambers, detector with scintillating optical fibers SciFi, proton recoil camera - CAMERA, and electromagnetic calorimeter - ECALO. The work on straw chambers embraced: high voltage supply distribution, coupling circuits with front end electronics, co-design of chamber mechanics, immunity of chambers to EMI, noise optimization of front end electronics. T2K (J-PARC to Super Kamiokande) is a long base 295 $\mathrm{km}$ neutrino experiment to confirm muon neutrino oscillation to electron neutrino. The team is responsible for time synchronization between two distant sites. The team was also involved in mass measurements and precise characterization of new generation of solid state photomultipliers of SIPM, MPPC or MAPD type.

X-ray, absolutely calibrated, spectrophotometer SphinX was constructed in CBK PAN Institute in Wrocław. The equipment serves for measurements of $\mathrm{x}$-ray emission from the Sun in the photon energy range $0,8-15 \mathrm{keV}$. The reference measurements are done by the X-ray monitors ZRM at GOES and NASA RHESSI satellites. SphinX was positioned in KORONAS-Foton satellite launched in 2009 from Plesietsk kosmodrome. Equipment technical data were: weight $4 \mathrm{~kg}$, power consumption max $10 \mathrm{~W}$, time resolution $6 \mathrm{us}$, energy resolution $1 \mathrm{keV}-15 \mathrm{keV}$, telemetry 150 $\mathrm{MB} / 24 \mathrm{~h}$. Spectrometer featured three detectors of various apertures and Be window. Construction was hybrid integrated in a TO package and included: detector chip, FET and preamplifier, temperature monitor, cooler and mounting stud. Laboratory and flight tests were done in a standardized way according to space requirements. The final measurement were done on the Sun's orbit. The SphinX results were compared with relevant measurements done by similar spectrometers RESIK, GOES, SOXS RHESSI and MESSENGER XRS. Now SphinX of the new generation is under design, basing on FPGA LEON3 microprocessor.

Photonic and electronic systems for space applications must fulfill stringent requirements concerning reliability. Research on such systems is carried out at the CBK - Space Research Center of PAS. One of the solutions is to use specialized integrated circuits which fulfill the requirements and are expensive. Other approach is to use COTS components but to understand extremely well their deterioration and proper work thresholds. Antifuse FPGA circuits are non-volatile one time programmable. They are accepted by ESA standards. The main challenges are: rising demand for photonics/electronics working in harsh conditions, need for standard reliability analysis methodology for complex circuit applications, need to compare fault mitigation techniques. Similar aspects are considered for applications in HEP experiments. The issue are SEE - single event upsets which are spoiling data quality accidentally, as differentiated with TID effects. SEE are dangerous because the errors propagate in data mesh/network and may affect also MO. The 
simulation system for these effects uses post place, route with incorporated time delays, and post back-annotate files and automatically replaces used target architecture primitives to their statistical models. Instead of Boolean, there is used statistical model of primitive use of probabilistic logic domain. Probability of SEE induced fault at primitive output level is modeled using manufacturers radiation characteristics radiation tests, and target model for radiation. In this way converted VHDL is processed in a classical way.

Electronic circuits, in critical applications like space technologies, may be configured partially against configuration as a whole entity. The procedure has three steps: define reconfigurable partition boundary, fit-check all modules, and generate. Proxy logics is done automatically, while decoupling logic is done by the user. The outcome is set of bit streams defining static, objects, modules, full and black boxes. Partial reconfiguration may concern functionality switching or extension of free resources. It may also concern malfunction prevention by implementing repair process, or isolating the faulty module. Experiments with dynamic partial reconfiguration was made using several processing units in various redundancy conditions at experimental PCBs. Experiments were done at real-life work conditions and timing constraints. DPR was employed for error injection, virtual fault generation and recovery. The test set-up consist of module were functioning or malfunctioning areas can be generated, repairable modules, nondependent modules from each other. Markov chains were used as tools for modeling the system. System is described in a form of finite states. Transitions between the states, and their rate, are well defined. There are used several different and nondependent system performance measures. Markov property is met by the modeled system. When the present state is known, the future process development is nondependent of anything that happened before. The aim of the procedures is to assure dependable work of a big system in such a way as to be at least one failure away from total system malfunction.

BRITE satellites are nano-satellites. BRITE is a constellation of six units where each satellite is equipped with only one telescope. Used technology is low cost, and short design time. The is no hardware redundancy, with the system life time only two years. Two of the nano-satellites are done at CBK PAN, together with local communication system at CAMK. The developments may be traced at BRITE-PL project web. Telemetry beacon for Polish payload on BRITE is done at CAMK PAN. The beacon works at $2,4 \mathrm{GHz}$ with $100 \mathrm{~mW}$ power, $\mathrm{CW}$ Morse code modulation. It does telemetry of chosen subsystems plus static beacon text. Main aims of the beacon subsystem are: to test low power satellite communication link, to assure radioamateur feature, to make Doppler position measurements possible.

\section{SYNCHRONIZATION IN DISTRIBUTED SYSTEMS AND PHOTONIC INTERNET}

Distributed systems (either photonic or electronics) usually need a kind of synchronization. There are numerous types of distributed systems of concern. The parameters and/or attributes of distributed systems are: geometrical size dimensions, connection distance, range/changeability of connection distance - centimeters on the PCB, meters between racks, kilometers between distant nodes, hundreds/thousand $\mathrm{km}$ in global systems; processing/user size - number of nodes - from 10 to $10^{4}$ or more; system nature - static or dynamic; system topology - star, tree-like, hierarchical, ring, lattice, mixed/hybrid; technology - optical, cable, wireless; synchronization - fully synchronized, partially synchronized, with synchronization islands, pipeline, non-synchronized, asynchronous. In some systems, like the best effort PC network, this synchronization is approximate. In deterministic communications channels the synchronization must be accurate. Even in non-deterministic system a quality measure QoS is introduced, which is a measure of synchronization quality. The stringent synchronization issues are met in distributed telemetric and control systems serving large research experiments in astronomy, astroparticle physics, high energy physic experiments, accelerator technology, free electron lasers and other big machine, where the time in particular parts of large object must be known precisely and synchronized to the experiment clock. There are several synchronization methods in distributed systems. There are also different synchronization types, requirements and goals. Different values are synchronized, like: frequency, phase, time offset, time order, succession of events, etc. Synchronization types are static or dynamic. The ultimate synchronization is distribution of atomic clock signals, what is required in some systems.

Phase-locked loop PLL technology is used frequently for synchronization purposes. The basic features of current PLL solutions are: they are doing full frequency synthesis, perform phase and/or frequency synchronization, there are multiple implementations of PLLs - digital and analog, they are crucial part of high speed FPGA chips. I large systems, or systems with complex structure, with many nodes, multiple signal sources, multiple clocks, etc, the issue is synchronization of multiple sources. In this case, synchronization may be done with respect to specific clock signal, selected signal source or dedicated FPGA processor, and its internal IO delay or PLL components. Synchronization of 
multiple sources includes: serial stream bit alignment, or multiple streams, data ID alignment including time-stamping and LUT delays; taking into account PVT drift, including such effects like process, voltages and temperature drifts and their influence on clock jitter - in long and short time horizons. A separate issue is synchronization in FPGA. Serial link synchronization includes: link training, setting of test patterns, determination of setup and hold time requirements, including FPGA specifics. These latter are: solving disadvantages combined with asynchronous logic, taking into account embedded CPU islands, varying place and route results, taking into account specific FPGA chip architecture requirements. Usually, in the systems of concern, the NTP protocol is not enough. NTP provides UTC, 20-70 $\mu$ s accuracy over public Internet, is hierarchical, semi layered, open source client and server software is available. PTP protocol is used instead, in demanding applications. PTP features are: provides sub-microsecond time precision, has mixed software and hardware support, exchanges effectively synchronization messages, operates with several different node types: as ordinary clock, transparent clock, boundary clock, main clock, grandmaster clock, etc. Extensions of PTP are researched. White Rabbit (WR) is one of promising extensions of PTP. It is researched at CERN and GSI for introduction in large experiments for accelerator machines synchronization. WR is an open design. It possesses sub-ns precision. Acts with dedicated synchronization channel on top of typical Ethernet network. Is back compatible with classical Ethernets. Specific precision synchronization system was developed for LHC accelerator infrastructure and its experiments. Timing, Trigger an Control system TTC has dedicated hardware and network structure. It is responsible for transmission of trigger and rigorously synchronized control messages. Each key board in the LHC and detectors has a TTC IO port. It acts with ns accuracy, because the LHC event clock in 25 ns. A proprietary ultra-precise synchronization system is under development to serve the FLASH and European X-ray free electron lasers. Synchronization is performed over optical links and RF channel. The system still under development offers from 50 to $100 \mathrm{fs}$ time jitter, and from $100 \mathrm{fs}$ to $100 \mathrm{ps}$ of drift. Active contributions to synchronization for E-XFEL, for LHC and other machines are generated by the research group PERG/ELHEP ISE at WUT. Components of the WR standard are also under development there.

A topical track on photonic Internet is a traditional subject of WILGA Symposia. It consisted of papers on photonic LANs, trunk optical lines of ultimate throughput, new optical non-blocking codes, developments in all-optical architectures. One of the concepts is usage of organic and polymer photonics in sensing networks. Application of organic photonics to build the framework of the variety of access networks to the terabit photonic transport network was considered with a potential to decrease costs of the system. Micro-structured plastic optical fibers - mPOF are under intense studies at UMCS and IMIO PW Institutes. They are cheap and are user scalable, even in singlemode version.

\section{FACILITY FOR ANTIPROTON AND ION RESEARCH (FAIR)}

The Jagiellonian University in Kraków has recently become a shareholder of the FAIR - Facility for Antiproton and Ion Research, a new large accelerator infrastructure under construction, located in GSI Institute in Darmstadt. Polish Consortium of FAIR includes also, apart from Jagiellonian University, Warsaw University, Warsaw University of Technology, AGH University of Science and Technology, Kraków University, National Center for Nuclear Research in Swierk, Institute of Nuclear Physics in Krakow. Total involvement is at the level of nearly 100 people, and financial involvement for the in-kind contribution is over $20 \mathrm{M}$ Euro. This massive involvement of the Polish accelerator R\&D community in FAIR is justified by several different reasons. First of all it is a world class facility of very big discovery potential, and attracts many scientists from around the world. Second is that there are no large accelerator facilities in this country of discovery potential. Third, FAIR in Darmstadt is very close to domestic facilities and local laboratories, where part of the FAIR work will be localized.

FAIR is going to be a really global initiative with partners from all over Europe and globally, including for example: Germany, France, Italy, Poland, Russia, Slovenia, Spain, UK, Saudi Arabia, and also very probably China, Ukraine, Brazil, South Korea, Japan, Turkey, the USA, and some others. FAIR will employ over 100 persons (FTE) including directly employed researchers, advisors and seconded. These numbers do not count all researchers expected to be involved in the experiments, and belonging to partners and FAIR related Collaborations. This number is expected to reach initially well over 2000, and finally well over 3000 persons. FAIR Research Division employment in 2020 is expected to reach around 300 persons. Percentage of the in-house researchers in FAIR is requested to reach the level of $12 \%$. This important indicator of the degree of laboratory internationalization is at other laboratories at the following levels: CERN - 6-9\%, DESY - 25\%, JLab - around 13\%, TRIUMF - 20-30\%, ESRF - 7\%. 
FAIR bodies are: Council - Scientific Council, A\&F Com., Machine Advisory Com., In-kind Review Board; Research Division - Expert Committee Experiments, Board of FAIR Collaborations; and Project Steering Board. The predicted FAIR experiments are: APPA - Atomic, Plasma Physics and Applications, CBM/HADES - Compressed Baryonic Matter, NuSTAR - Nuclear Structure, Astrophysics and Reactions and PANDA - Antiproton Annihilation at Darmstadt. This large development is realized in modularized version to enable more efficiency in civil engineering and work progress. APPA and PANDA is associated with HESR, APPA with SIS 100, NuSTAR with RESR. The civil construction includes: over $1 \mathrm{~km}$ of synchrotrons, 0,6 km HESR ring, accelerators with beamlines over $3 \mathrm{~km}$, usable area nearly $15010^{3} \mathrm{~m}^{2}$. Total finances of the project are over 1 billion Euro. Radiation protection licenses for the new infrastructure are under process by relevant authorities. The roadmap for civil engineering constructions predicts major civil construction infrastructure readiness by the end of 2016. Installation and commissioning of accelerators and detectors take place during 2017-18. Data taking is expected to begin at the end of 2018. FAIR is expected to gain its full nominal luminosity in 2020. FAIR operation costs will be shared by the shareholders proportionally to their financial input and to the expected infrastructure usage. Current estimates (rough) of the annual operational costs of FAIR are around $200 \mathrm{M}$ Euro in 2013 prices.

FAIR is an essential extension of the exiting GSI facilities. Actually it outgrows the old infrastructure very much. Proto beams are pre-accelerated in the injector and accelerator in proton linear accelerator - p-LINAC. Heavy ions are accelerated in the U-LINAC. Proton and heavy ion beams are fed into small ring SIS18. Then, the beams are directed to the new infrastructure, bigger rings SIS100 and SIS300 (if there i a need for further beam acceleration and processing). The protons are used also to produce antiprotons, by converting them into a special dedicated production target. Proton beam may be also used directly for experiments in the APPA facility. On target produced antiprotons have to be collected into the beam and the bunches again, and then accelerated to relevant energies. Antiprotons are gathered in the collector ring CR and RESR ring. Ready, high quality bunched antiproton beam is injected into HESR. HESR ring carries PANDA experiment. High energy heavy ion beam are directly used to collide with steady target in the CBM experiment. They may also be used for production of exotic unstable ion beams. Unstable ions are produced in the Rare Isotpe Poduction Target and filtered the Super-FRS, where the NuSTAR experiments will be run.

FAIR assumes many new solutions in accelerator technology, breaking a number of technical challenges. Building compact and cost effective accelerators require application of fast cycling superconducting magnets with the field changeability reaching $\mathrm{dB} / \mathrm{dT}$ around $4 \mathrm{~T} / \mathrm{s}$. Fast acceleration of the beam requires innovative cavities of high gradient, variable frequency, ferrite and MA loaded. Applied extremely high voltage and resulted high beam intensities require equally extreme high vacuum. The requirement for the vacuum is over $10^{-12} \mathrm{mbar}$. Ultra-precision beams are required by the FAIR very demanding experiments. The innovative techniques like electron and stochastic cooling will be used for the beam quality and precision improvement. Full set of superconducting magnets are under construction, including SIS100 main dipole magnets (coils production by BNG), SIS 100 precision sextupole magnets (DUBNA prototypes are tested). The HESR layout includes beamwise: two straight sections and two bending sections, $p$, pbar injection from CR RESR; RF accelerating cavities, PANDA, stochastic pickups, bending magnets, stochastic kickers (connected by SC signal paths with stochastic pickups) electron cooler, helix dipole magnet, and bending magnets. The main machine parameters are: momentum range $-1,5-15 \mathrm{GeV} / \mathrm{c}$; circumference $-575 \mathrm{~m}$; magnetic bending power $-50 \mathrm{Tm}$; dipole ramp $25 \mathrm{mT} / \mathrm{s}$; acceleration rate $-0,2(\mathrm{GeV} / \mathrm{c}) / \mathrm{s}$; geometrical acceptances for $\beta_{\mathrm{t}}=2 \mathrm{~m}$ - horizontal around $5 \mathrm{~mm} \mathrm{mrad,}$ vertical around $6 \mathrm{~mm}$ mrad; momentum acceptance $\pm 2,3 \times 10^{-3}$. The following HESR components are prototyped and tested at COSY: pellet target, WASA, barrier bucket cavity, stochastic cooling, $2 \mathrm{MeV}$ e-cooler, and residual gas profile monitor. APPA experiment irradiates sample with annular ion beam. The interaction region consists of tmper shell, beam heated region, payload, sample material in which shock wave propagates causing protons ejection to HEPW detector. CBM is an experiment of QGP quark gluon plasma research with energetic ion beam and steady target. The other two experiments are PANDA and Super FRS, NuSTAR. All experiments prepare their own detailed TDR documents. Also other relevant and required legal documents are generated including: timelines for experiments, MoUs, risk assessment, etc.

\section{ELECTRONIC/PHOTONIC SYSTEMS FOR COMPRESSED BARIONIC MATTER (CBM) RESEARCH}

CBM is currently preparing its TDR document. CBM Collaboration has now over 150 members. Around 50 Ph.D. students are associated with the work planned at the CBM experiment and facilities. This number of young researchers 
affiliated to CMS is astonishing, and shows extremely vivid character of the research field, attracting a lot of interest. Hardware and software developments for CBM are underway. CBM is exploring the QCD phase diagram. QCD phase diagram has coordinates - temperature $\mathrm{T}[\mathrm{K}]$ and baryon chemical potential $\mu_{\mathrm{B}}$. Hadronic phase is observed for low $\mathrm{T}$ and low $\mu_{\mathrm{B}}$. The QCB phase diagram is expected to have such critical points and lines (analogous to classical phase diagrams) like liquidus, solidus, etc. Hadronic phase may contain an equilibrium point for gas - liquid. A nuclear superfluid phase is expected to be observed. Critical point (points) divide the phase lines to expected nuclear superfluid, quarkyonic matter, chiral density wave, possibly even crystalline quarkyonic states, crystalline CSC states, 2SC state, CFL state, and color superconducting phases, etc. There are two essential approaches to research QGP - high T and small $\mu_{\mathrm{B}}$, and vice versa, small $\mathrm{T}$ and high $\mu_{\mathrm{B}}$. The first case - high $\mathrm{T}$ and small $\mu_{\mathrm{B}}$ is relevant to the early universe. Baryon chemical potential was close to zero then. An additional phase is expected there of sQGP. The following experiments are able to research this region: ALICE, ATLAS, and CMS at LHC, as well as STAR, and PHENIX at top RHIC energies. Probing the QCD diagram at moderate T and very high $\mu_{\mathrm{B}}$ is available for the following experiments: beam energy scan at RHIC, NA61 at CERN SPS, CBM at FAIR, and MPD at NICA.

Quark matter is expected to exist in stable phase in massive neutron (or quark) stars. It is also expected that depending on the mass there are observed hadron states, mixed quark/hadron phase and pure quark matter. CBM research program includes many potential observables. SIS100/300 is able to observe the excitation function of the collective flow of hadrons which is driven by the pressure created in the early fireball. The observations at SIS100 include the excitation functions of multi-strange hyperons in $\mathrm{Au}+\mathrm{Au}$ and $\mathrm{C}+\mathrm{C}$ collisions at energies from 2 to $11 \mathrm{~A} \mathrm{GeV}$. The electromagnetic radiation emanating from the fireball and chiral symmetry restoration in dense baryonic matter are measured in $\mathrm{Ar}+\mathrm{KCl}$ and $\mathrm{Pb}-\mathrm{Au}$ collisions. Phase transitions are to be observed from hadronic matter to quarkyonic or partonic matter at high net baryon densities. Hypernuclei are expected to be created, as well a strange dibaryons and massive strange objects. Production of hypernuclei (single and double) takes place via coalescence of hyperons and light nuclei. Trange dibaryons and heavy multi-strange short-lived objects may be generated. Such collisions are good field for charm production. Charm production mechanisms will be researched, as well as charm propagation, and in-medium properties of charmed particles in dense nuclear matter.

CBM technical developments are underway, including: SC dipole magnets, precision magnets, Micro-Vertex Detector, Silicon Tracking System (STS), MRPC ToF Wall, RICH Detector (Ring Imaging Cherenkov), Muon Detector, Transition Radiation Detector, Forward Calorimeter, DAQ and online event selection. The role of the micro-vertex detector (MVD) is to determine the secondary vertices of open charm decays for times $10^{-12}-10^{-13} \mathrm{~s}$. Monolithic active pixel sensors MAPS were applied (solid state photomultipliers). Technical data of MVD are: square pixel size $20 \times 20 \mu \mathrm{m}^{2}$, position resolution $4 \mu \mathrm{m}$, vertex resolution $500-100 \mu \mathrm{m}$ along the beam axis; total material budget silicon equivalent $300-500 \mu \mathrm{m}$, three fully equipped /detector/measurement stations $-5,10$ an $15 \mathrm{~cm}$ from the target. Prototype RICH beam station test at SERN-SPS is under construction at several CBM partners including Giesen, Wuppertal,etc. Beam tests are done in CERN with the prototype of the following CBM detectors: RICH, TRDs and RPCs. TRD requirements are: $100 \mathrm{kHz} / \mathrm{cm}^{2}$ and $700 \mathrm{~m}^{2}$. The load test of full size prototype MPRC TOF Wall is done at GSI. There are done simulations for high speed analysis with STS and ToF particle identification. The assumptions are: realistic geometry, material budget and detector response. ToF at $10 \mathrm{~m}$ is measured with resolution of $80 \mathrm{ps.} \mathrm{CBM}$ simulations are done with central $\mathrm{Au}+\mathrm{Au}$ collisions at different energies. Hyperon yields are calculated. D-meson production is awaited and observed at SIS100 and SIS300. Simulations are done for Dimuons from central $\mathrm{Au}+\mathrm{Au}$ collisions at different energies.

IS100/300, CBM has a potential to research the QCD phase diagram in the region of high baryon densities. CBM has extreme potential in high precision measurements of multi-differential observables including particles with small cross sections for a variety of beam energies and collision systems $\left(\pi+-, \eta, \rho, \omega, \varphi, \mathrm{K}^{+-o},, \mathrm{p}, \mathrm{p}, \Lambda, \Lambda, \Sigma^{*}, \Xi+-, \Omega+-, \mathrm{D}+-0, \mathrm{~J} / \psi, \psi^{c}\right)$. The constructed prototype detector performances fulfill CBM requirements. TDR documents are in their close to final versions.

\section{MONOLITHIC ACTIVE PIXEL SENSORS (MAPS) AND VERTEX DETECTOR FOR CBM}

MAPS detectors are developed and increasingly frequently used for HEP applications as vertex detectors. They were applied at IPHC in Strasbourg in 1999. Now they are applied in possible ITS upgrade in ALICE, STAR experiment, ILC collider and at CBM, FAIR GSI. MAPS matrix avalanche photodiodes (solid state photomultipliers) are used by 
industry in specialized digital cameras. Have been modified for charged particles detection for IPHC usage. Since then they are developed by the above mentioned collaboration with shared costs. MAPS are now also manufactured by HAMAMATSU. The development directions for these components are: increase single point resolution, direct binary readout, better material budget, improve radiation hardness to non-ionizing radiation, as well as to ionizing radiation, improve time resolution, introduce massive parallel readout. Some of technological optimizations include: thinning the active layer of component, pixel pitch optimization and use high resistivity material, thermal annealing of radiation defects. The structure of MAPS is: $p+$ back side, $p$ - substrate (epitaxial layer), $n+-p+$ diode pixel - $p+$ layer and $n+$ localized pixel ( $\mathrm{p}$-well structures), $\mathrm{SiO}_{2}$ insulation cover with contact only to $\mathrm{n}+$. The simple operation principle of MAPS is that passing particle induces charge in the epitaxial layer. To be fully relevant for HEP applications as vertex pixel detectors these components have to be resistant to the non-ionizing and ionizing radiation. For non-ionizing radiation the following effects are observed in MAPS: the energy i deposited into the crystal lattice, atoms may be displaced, the effects are caused by energetic heavy charged and neutral particles like fast leptons and hadrons. For ionizing radiation the following effects are observed in MAPS: energy is deposited into the electron cloud, radiation can ionize atoms and destroy molecules, the effects are caused by charged particles and photons. Destruction of the structure of epitaxial layer and pixel well layer increases the number of defects localized there. The noise increases and leakage current. Some of the harmful effects may be reversed by appropriate thermal annealing. Thermal annealing is usedto suppress ionizing damage without drawbacks of reverse annealing. Component cooling is used as a measure against non-ionizing radiation. Noise is alleviated by several times with decreasing temperature in MAPS. Defects due to radiation reside mainly in the epitaxial layer. Charge losses there originate due to recombination at the radiation induced defects. There is a difference in the influence of radiation damage between MAPS of large and small pixel pitch. In both cases the whole generated charge is detected. There are however smaller losses for large pixel pitch, even un-irradiated. Generally, in the MAPS (irradiated and un-irradiated) the signal losses depend on the pixel pitch. Small pixel pitch improves radiation tolerance, expressed in $\mathrm{n}_{\mathrm{eq}} / \mathrm{cm}^{2}$. Radiation tolerance was measured between $10^{11}$ and $10^{14}$ $\mathrm{n}_{\mathrm{eq}} \mathrm{cm}^{-2}$. Pixel pitch is changed in the range $10-40 \mu \mathrm{m}$. The drawbacks are as follows. With the increasing number of pixels in the component the readout time is considerably larger and power consumption of the components is larger. Decrease of doping concentration in epitaxial layer increases the CMOS component resistivity. Typically the resistivity is around $30 \Omega \mathrm{cm}$. High resistivity component has $1 \mathrm{k} \Omega \mathrm{cm}$. Components of high-resistivity have larger depleted volumes, which results in accelerated charge collection and improved non-ionizing radiation tolerance. Neutron irradiation of MAPS damage parasitically the surface of the component. This increases the noise of the component. This noise contribution may be alleviated by thermal annealing. MVD prototype MAPD sensor, called MIMOSA, was manufactured by the Goethe Uni. Research team involved in CBM. Mimosa was researched to be immunized to radiation, non-ionizing and ionizing. Ionizing radiation hardness was improved by changing the CMOS technology from 0,35 to $0,18 \mu \mathrm{m}$. Thermal annealing was used to suppress ionizing radiation damage effects.

The work on CBM-MVD is done by a group at Goethe Uni. Frankfurt am Main. Beam tests are done at CERN. The role of MVD at CBM is: improve secondary vertex resolution; background rejection and di-electron measurements. The construction of MVD hosts highly granular silicon pixel sensor featuring fast read-ott characteristics, excellent spatial resolution and robustness to radiation environment. The required performances for MVD at SIS-100 are: radiation tolerance bigger than $10^{13} \mathrm{n}_{\mathrm{eq}} \mathrm{cm}^{-2}$ and bigger than $1 \mathrm{Mrad}$; read-out speed bigger than $30 \mathrm{kframes} / \mathrm{s}$; intrinsic resolution smaller than $5 \mu \mathrm{m}$. Prototype of the MVD was fabricated with framework, sensors, CVD diamond substrate, Al heat sink and front end electronic boards FEB. High resistivity Mimosa sensors were used in the prototype. The dimensions of sensors with integrated electronics are approximately $10 \times 20 \mathrm{~mm}^{2}$. The issues to be solved were: mechanical integration, DAQ and data analysis.

\section{HIGH PERFORMANCE COMPUTING AND COMPUTATIONAL INTELLIGENCE BIOMEDICAL SYSTEM DESIGN}

Statistical learning systems is a paradigm for designing classifiers based on data. Statistical classifier is a hyper-surface separating points corresponding to various classes. There are several learning methods for such systems: supervisedwith multi-layer perceptron and kernel machines; semi-supervised - with transductive inference, or how to use information contained in the distribution of labeled points and the clusters of unlabelled data; unsupervised - based on structure of clusters and self-organizing maps. The work on such high performance is carried out by the WUT ISE team chaired by prof.S.Jankowski. Semi supervised learning systems promise decrease of calculation effort and not reducing too much of the advantages of unsupervised learning systems. Semi-supervised systems should make use of the most 
relevant information of labeled points. Influential statistical analysis of learning systems is applied for weighting the influence of each data point by comparison of system properties before and after leaving this point out of the learning set - the leave one out residuals. The primary aim is to reduce computational complexity what involves excess costs. LS SVM is used as a statistical classifier in combination with virtual leave-one-out procedure. In summary, semisupervised lease squares support vector machine is a statistical learning system that explores the information from the labeled data a well a unlabelled data distribution in the input space. It is an extension of supervised least squares SVM. The issues for efficient algorithms are: find simple solution for the supervised learning, define a smart criterion for the selection of the most influential unlabelled points in order to perform the label switching test. After tests it turns out that the virtual leave-one-out method enables obtaining estimated values of the leave-one-out for the entire training set in a single step with no retraining required. For the semi-supervised LS-SVM the calculations can be expressed in analytic form.

One of the basic tasks of computational intelligence systems is processing large amounts of data, which are nor readable by humans, and transferring them for human usage. This includes for example implementation of fully automated monitoring and reporting systems. The tasks are to extract the most significant information from the collected data and generate short reports, only if needed. The involved processing is smart data compression. The system requirements are (not all to be fulfilled at a time):compatibility with human perception, images, high abstraction level of the output reports like fire/smoke in room, unauthorized entrance, human like perception, ability of abstract reasoning, etc. Smart data compression includes also the ability to send the reports via arbitrary channels, including slow ones like SMS. System assumptions are not based on deterministic actions, thus, preprogramming is excluded, but adaptive methods are preferred. Other assumptions are: software modeling the behavior of nature, animals and humans, the primary input data are not important, but the processing results, the operator has no right to trigger any alarm but the machine. To solve this issue the restricted Boltzman machine was used. RBM is one layer stochastic artificial neural network (NN) which has unsupervised learning ability, and is based on relatively simple mathematical model. The system was tested initially for handwriting recognition.

Numerable solutions of computational intelligence systems rely on neural networks (NN). Some of the neural networks may be realized in photonics technologies. There are passive and active methods of NN learning. Effective active learning methods are based on various kind of statistics. Frequently used statistic is PRESS - predicted residual sum of squares statistic - validation how good the model fits the observations. NN model may be improved to minimize the generalized error. Improvement criteria selects experiments to reduce the number of needed calculations and their associated costs. The task is, at given training set, to define the criterion for the model selection. The best architecture is selected by controlling the over-fitting, and next out of this architecture the best model is selected. The basis of active learning is to define a methodology to select the best experiments from a candidate set and to add them to the training set. Generally, NN learn by adapting the weights of their synaptic connections to the changes of the surrounding environment in which and for which the NN works. It is to be remembered that the NN works always in noisy environment and the information around has probabilistic character. Only in such environment, the NN should be able to generalize the examples. NN works with multi-layer perceptrons, includes hidden units, and the activation is by sigmoid function. Problem generalization includes bias-variance dilemma. Optimal number of coefficients is chosen for the network to obtain the best generalization of data. The generalization error is decomposed into a sum of two components bias - inversely proportional to the NN complexity, and variance - proportional to the NN complexity. Model selection via leverages used for nonlinear models assumes that each observation has influence on model parameters. Virtual leave-one-out approach is used and the error is predicted when the example is virtually removed from the training set. The procedure selects the model out of models of the same architectures and selects the optimal architecture. Back-propagation method is used when the NN produces incorrect output response. Back-propagation enables calculation of the error derivatives with respect to the weights, and the $\mathrm{n}$ discover which hidden units are responsible for error generation and to determine which weight to adjust and by how much. Virtual leave-one-out uses Jacobian matrix and leverages. Supervised learning systems have to be extensively trained to perform well. This involves thousands of experiments. Usually the system is resource limited and cannot collect big data amounts. Experiments have to be selected efficiently to minimize their number. Active learning methodology is: train NN with examples, compute estimated leverage for candidates, select the example with the leverage, add the new example, compute again till the criterion. 
Some of the computation problems are resolved efficiently by means of graph NN. Examples are represented as graphs, and the used tools are graph and node classifiers, multifold cross-validation, sub-graph matching, building of contraction map based on Banach theorem, penalty based on contraction constant, quality estimates based on quality precision recall, penalty imposed on weights when the contraction is lost, calculated GNN and FNN values, boundary values depend on dataset, etc. Most graphs can be used including non-positional and cyclic.

Support vector machine is an efficient classification tool used in NN. NN SVN are based on discrimination in multi dimensional feature spaces. The training sets are points in feature space. These points represent actually vectors of fixed length. The assumed similarity measure is based on distance between points in the feature space. This approach is only valid for cases when data can be represented a vectors of fixed length. Data are frequently structured and represented as graphs, especially in biological systems, where we classify molecules, images, etc. Feature extraction from such data turned out not to be effective for simple methods of data classification. These include physical or chemical properties, to be chosen as features. Immediately the number of data is overwhelmingly large. What is the alternative to full data analysis? It is also believed that important information is encoded in the structure, distribution of points and graphs based on them. The information is encoded in connections and relations. This information is disentangled buy using recursive distributed representation method with usage of labeling recursive auto associative memory LRAAM. The method generates vector representation for graphs and enables effective use for computational classification and approximation tasks. Feed-forward N (FNN) is trained to perform auto-association. The training sets are nodes of the graph including labels and pointers to adjacent vertices. The labels may contain additional information about the node. This information may be physical or chemical properties. The outputs of hidden neurons are viewed as the compact representation of the input node. Pointers representation is obtained in the training process. The process is initialized with random values. Any suitable gradient descent algorithm, like Levenberg-Marquardt, is used for learning. To address non stationary problems, called moving target problem, a part of the training set changed during the process. However, the convergence of the training procedure is not guaranteed. Thus, the training procedure is very sensitive to learning parameters and as a result pointers showing different structures obtain different representations.

A lot of research and design work on new biomedical components and systems are carried out at the Faculty of Mechatronics WUT. Some of these solutions ate developed in collation with research clinics and specialist physicians. One of the running projects is digital stethoscope for cardiac auscultation. Physiological acoustic heart sound is standardized to four periods $\mathrm{S}_{1}, \mathrm{~S}_{2}, \mathrm{~S}_{3}, \mathrm{~S}_{4}$ and murmurs. Spectrum of this sound is $50-500 \mathrm{~Hz}$. The strongest, and audible are sounds 1 and 2. Digital stethoscope device consists of sensor, pre-amplifier, band pass and denoising filter, ADC, software, data recording, data processing in MatLab, and display. Classical stethoscope was equipped with integrated digital microphone and data transmission link. Signal processing tasks are: denoising, improve the signal to noise ratio, features extraction (using wavelet transform), automatic signal segmentation, classification between normal and abnormal.

Artificial patient modules of many kinds are researched for the purpose of testing and development of biomedical apparatus. The modules can be of general multipurpose character or narrowly specialized for testing of particular hardware, software, or new measuring method. Here, an artificial patient module was build for testing of bio-impedance measuring devices (prof.G.Cybulski). Bio-impedance measurements are used to record or monitor: cardiovascular system activity, respiratory system activity, thoracic fluid content, and body composition. The interface between skin and electrode is modeled by assuming an electrical equivalent circuit, in a form of passive RC circuit containing two resistors and a capacitor. In order to produce small changes of tissue impedance with high precision, and imitate tissue impedance as good as possible, two resistors were used, one constant and one variable. Constant value of tissue impedance is much greater than amplitude of variable component. Artificial patient was designed as a connection of two circuits modeling electrode-skin interface and two resistors simulating tissue resistance. Electrode potentials are imitated by batteries a voltage sources connected in series with relevant resistor. The system was tested for chosen impedance waveforms close to physiological changes. Various waveforms of impedance changes were programmed and tested, which relate to different influences on tissue impedance. This specialized artificial patient may be used to verify and calibrate various bio-impedance measuring devices.

The effect of body weight and posture on acceleration of platform vibrating plate is researched by the same team as above. Mathematical model of human body is developed to describe its interaction with the vibrating platform. The 
research concerns the methods of vibration training WBV - whole body vibrations method. The advantages of WBV are: increase of skeletal muscle strength and bone density, improvement of balance in sub-populations prone to fail, and safe alternative to resistance training, alleviating in chronic back pain. The negative effects of vibrations are: organ resonance, where the resonance frequencies of the selected human organs are in the range of $4-10 \mathrm{~Hz}$ for chest, abdomen and spinal organs. Safety precautions prompt to use frequencies above $20 \mathrm{~Hz}$. The tests were doe on students in three positions: light squat, deep squat and squat with heels raised. The used vibration plate was standardized for medical examinations. It was shown that posture evidently influence the experiment conditions by changing the max acceleration. Raising heels increases acceleration of the vibrating plate. The mathematical model of human body and the platform should take into account the influence of the body posture on vibrations andgradual decline of acceleration from lower limbs to head.

The Microsystems and Sensors Research Group at ISE PW is carrying out broad spectrum of works also on sensors and sensory systems for biomedical applications (prof.R.Jachowicz, prof.J.Weremczuk). Main specialty are humidity sensors. Dew point hygrometer was performed in integrated MEMS type version, using either optical or impedance detection. The advantage is ultra fast action of the component. Dew point MEMS consists of detector, thermo resistor, neater and contact pad. Semiconductor version of the detector was also manufactured. Associated numerical calculations include modeling and then optimization of temperature distribution in the micro system, water molecules and heat transport modeling, with the aim to build and verify the full hygrometer model, optimize hygrometer dynamics. A number of different microsystems were designed and manufactured for medical applications. Fast hygrometer for laryngology was tested in Military Medical Institute in Warsaw. Dermatological applications include TEWL factor measurements (Transepidermal Water Loss). Hygrometer for TEWL measurements was applied in cosmetology. Other research subjects of the Group include: wireless interfaces for passive microsensors, microsensor packaging, passive digital interfaces, ink-jet printing technology for microsensors, flexible sensors printed on Kapton foil and on textiles, THz range microsensors, smart sensor networks for buildings and environmental measurements, Microsystems for UAVs, .

Biomedical and socio-medical data are sensitive and have to be treated specially. Research work on mass data acquisition and aggregation in socio-medical studies and trials is done at ISE PW in cooperation with Medical University of Warsaw (dr Z.Wawrzyniak). The system includes the following functionalities, with arguments: data management (what for, how to do it, where ad when, who), e-health medical applications requirements (requirements, diseases, patients, medical devices), data mobile communication (systems, devices, protocols, software). The field of some e-health paradigms includes: tele-health, tele-medicine, m-health, remote patient monitoring, mobile patient monitoring and many more. The requirements for data research in the area of socio-medical trials are: sources of data (spatial and temporal), heterogeneity and inconsistency, manual vs machine made, data and system scalability, variability (numbers and categories), security and privacy, adequate ICT solutions, and staff resources. Trials by their purpose are divided to the categories: prevention, screening diagnostic, treatment, quality of life, compassionate use. Statistical requirements for the trials are: sample size, randomization, blinding; study protocol and possible needs for a pilot study; type of statistical analysis - explanatory, factorial; design of questionnaires and clinical report forms; data collection; construction of data coding schemes; data entry, database, data retrieval; and data validation, QA schemes. The flow of processes from raw data to knowledge includes: patients data input, pre processing, filtering, validation, analysis and classification, predictions using models, information output visualization and knowledge. A number of medical trials were done in Poland using these procedures. These included (2005-2012): ECAP - epidemiology of allergic disease; GAT - global adult tobacco survey; PONS - infrastructure for population study of health. The main features of central database server are: all data in a singe place, available immediately, electronic versions of questionnaires, reduction of pollster mistakes, central administration, time stamps on every document, export to text files, system of periodic reports.

\section{DNA COMPUTING}

DNA assembler is researched for next generation sequencers. The aims are: to reproduce complete genome sequence, at the conditions where chemical and physical processes produce shotgun reads and randomly extracted fragments need to be put together to reproduce original DNA sequence. Reads come from random positions and oversampling is necessary. Next generation sequencing features much lower costs, better throughput, what results in shorter reads. Oversampling must be higher and the amount of input data increases. To handle this weighted De Bruijn graph 
technique is applied. There are several difficulties to handle: complementary base pairing - reads can come from both, base or complimentary strands; solution is to build double graph; each read is considered as two reads - original and complementary; assembler should find two euler paths, which could be complimentary; random reads do not cover sequence evenly and correct edge weight is critical; solution is to normalize graph - divide each edge weight by average edge coverage; some wrong values are automatically repaired. The application architecture is designed as server client. Client is a web application.

DNA molecule logic gates are the base for standardization of DNA computing process. In the DNA computing systems, a signal is represented by a DNA string. This string may be changed to optical and/or electrical signals. The signals obey strict rules of DNA sequences. A single-stranded DNA string has a phosphor-sugar backbone with two different 5' and 3' ends and four bases - Adenine, Thymine, Cytosine and Guanine, denoted by ATCG. The individual genes are concatenated. Amplification PCR and exo-nuclease activity is observed. Polymerase may be cloned. DNA computing is interested in DNA sequence modification. Only polymerase gene part responsible for polymerase activity is cut out of the whole Taq polymerase gene. The rest of polymerase peptides are lost. DNA structure undergoes evaluation. The amplified product is put in plasmid vector, a circular DNS molecule, and cloned within bacteria colonies inside cells in order to reconstruct DNA structure which is more suitable for living cell environment, with all methylase DNA protection against restriction enzymes. The next stage is polymerase generation. After retrieving the new DNA bacteria plasmid clones and it is placed in an expression vector. The protein obtained from the next bacteria is just the modified Taq polymerase. Molecular OR gate is created. A signal equal to zero is in the case when a particular signal molecule is not present in the solution. In a process of output signal generation, any input signal, out of three, has to be present. This means than each signal can be a primer in the amplification reaction, which leads directly to the generation of the output signal. Extending these processes leads to the lab-on-a-chip DNA reactor. The work on DNA computing is carried out at ISE WUT in prof.J.Mulawka Group. This reasoning and experiments show that it is possible to implement expert rule inference based on molecular logic gates even in the lab-on-chip under automatic control. The applications of the method are interesting, like: detection of genetic diseases, gene connections in computer controlled reaction systems, very efficient processor for binary numbers.

\section{IMAGING TECHNIQUES}

Unmanned aerial vehicles and systems are used in diverse fields, wherever inexpensive and easy to deploy platforms are required. Small UAV, able to fly in easy and safe autopilot mode, is able to do valuable aerial surveys of archeological sites. A smart, $1 \mathrm{~m}$ octo-copter was done by a group of students from La Sapienza, Univ. Sassari and ISE WUT. The UAV has autopilot system with preloaded flight plan. Plan includes auto takeoff, landing and waypoint navigation. The avionics does accurate 3D aerial scanning, measurement and surveying in archeological sites. The methodology includes: flight planning, sensors setting-up, images processing, orto-photos or 3D model building, automatic extraction of features and classification. Cost confinements for such solutions impose the choices for small drones, of small payload up to $20 \mathrm{~kg}$. Due to little space in the avionics bay it should be used very efficiently. Image based 3D modeling is applied as software platform for the archeological missions of the UAV. The 3D model is obtained from photogrammetry based on aerial image pairs. Using standard camera and lenses, the system has $\mathrm{cm}$ resolution on the ground. The resolution measure is corresponding pixel dimension on the ground and observed ground swath - along and cross. Commercial expensive and much bigger laser scanners have only sub-cm resolution, for this distance, which is not much better. The observed ground swaths (along and cross) were in the range of 100-200 m. The surveillance results help understand and document the dynamics of the archeological deposit formation. The results obtained from small UAV photogrammetric procedures, replace perfectly ground based measurements, enable easy multi point triangulation, provide image-based modeling, and 3D modeling. The imaging tests along the line of UAV flight were done for the following parameters: flight height $100 \mathrm{~m}$, flight speed $6 \mathrm{~m} / \mathrm{s}$, sequenced trigger making one image per second, adjacent image FOV overlapping in the flight direction - more than $80 \%$. Practical tests were done with real archeological objects. Proper flight planning assures that the necessary flight is very short, takes only below 10 minutes, even for the objects of hundreds of $\mathrm{m}$ or single $\mathrm{km}$ size. Relevant number of pictures is taken. The programmetric software calculates camera position for every image. A dense cloud of points is generated, meshed and textured to result in true-color 3-dmensional model. The model is very accurate. Measurable and photorealistic or textured. The UAV in archeological applications enables planning improvement of excavation, saves time and money. 


\section{PHOTONICS AND ELECTRONICS APPLICATIONS}

The main topical tracks of WILGA Symposium concern photonics applications. A number of session concerning the applications of photonic and electronic circuits and systems included work on particular engineering and technical solutions for various fields like: astronomy, car industry, airborne industry, robotics, management of the road traffic, remote control methods for utility systems via the Internet, audio and video techniques, biomedicine, safety techniques, home appliances. A group of work concerned the development of a mobile platform for a universal robot equipped with advanced devices like cameras, grippers, Other group concerned the development of distributed measurement networks for minimum energy service of the network of self configuring environmental sensors. These sensors are expected to use a lot of energy harvesting. Some of these works are result of local and national photonics projects financed by local and national funding agencies. Photonics is more and more considered in this country as an enabling technology in many fields of national economy.

\section{CONCLUSIONS AND WILGA 2014}

The WILGA May 2013 meeting was a fruitful event gathering young researchers from the fields of advanced photonics and electronics systems. The 2014 Symposia on Photonics and Internet Engineering will be held on 23-25 January 2014 at WEiTI PW building in Warsaw and on $26.05-01.062014$ in Wilga Resort by PW. The organizers warmly invite young researchers to present their work. The WILGA Symposium web page is under the address:

http://wilga.ise.pw.edu.pl. WILGA Symposium has produced more than 2500 articles, out of which over 1000 were published in Proc.SPIE [2-4]. Several hundred of them are associated with the research activities of the PERG/ELHEP Research Group at ISE WUT. The Group is an initiator and major organizer of the WILGA Symposia.

\section{ACKNOWLEDGMENTS}

The author would like to thank all participants of WILGA Symposium for making the event again and again a success.

\section{REFERENCES}

1. WILGA Symposium web site: http://wilga.ise.pw.edu.pl

2. Proc. SPIE 8454, WILGA 2012

3. Proc. SPIE 8008 , WILGA 2011

4. Proc. SPIE7745, WILGA 2010 